TITLE:

\title{
Spectral diffusion of neutral and charged exciton transitions in single CdSe/ZnS nanocrystals due to quantum-confined Stark effect
}

\author{
$\operatorname{AUTHOR(S):~}$ \\ Ihara, Toshiyuki; Kanemitsu, Yoshihiko
}

\section{CITATION:}

Ihara, Toshiyuki ... [et al]. Spectral diffusion of neutral and charged exciton transitions in single CdSe/ZnS nanocrystals due to quantum-confined Stark effect. Physical Review B 2014, 90(19): 195302.

ISSUE DATE:

2014-11

URL:

http://hdl.handle.net/2433/191100

RIGHT:

(C)2014 American Physical Society 
PHYSICAL REVIEW B 90, 195302 (2014)

\title{
Spectral diffusion of neutral and charged exciton transitions in single CdSe/ZnS nanocrystals due to quantum-confined Stark effect
}

\author{
Toshiyuki Ihara and Yoshihiko Kanemitsu \\ Institute for Chemical Research, Kyoto University, Uji, Kyoto 611-0011, Japan \\ (Received 19 July 2014; revised manuscript received 2 October 2014; published 4 November 2014)
}

\begin{abstract}
Photoluminescence (PL) fluctuations of single semiconductor nanocrystals (NCs), such as PL blinking and spectral diffusion (SD), reflect the quantum nature of charges in the NCs. Through simultaneous measurements of PL spectra and lifetimes on single CdSe/ZnS NCs, PL of neutral excitons is found to exhibit a unique behavior of $\mathrm{SD}$, which is accompanied by changes of radiative recombination lifetime. We find that the SD of neutral excitons originated from the quantum-confined Stark effect, which also affects the SD of charged excitons observed during PL blinking.
\end{abstract}

DOI: 10.1103/PhysRevB.90.195302

PACS number(s): 78.67.Bf, 42.50.Ar, 78.55.Et, 78.67.Hc

\section{INTRODUCTION}

Spectroscopy on single semiconductor nanocrystals (NCs) has enabled us to reveal the quantum nature of charges in spatially confined systems at room temperature [1,2]. Individual NCs usually exhibit photoluminescence (PL) profile instabilities, such as unstable PL intensity (known as PL blinking), and irregular PL transition energy [known as spectral diffusion (SD)] [3-9]. PL blinking, the term used for time-dependent digital jumps of the PL intensity between on and off emissivity states, has been attributed to the charging of the NCs or the activation of trapping processes in impurity states [10-19]. In contrast, SD, which is an unstable fluctuation of the PL transition energy, has been attributed to the quantum-confined Stark effect (QCSE) [6-9,20-25]. Since these PL profile fluctuations prevent us from developing stable, single-photon emitters for application in quantum communication and cryptography $[26,27]$, the understanding and the control of those PL fluctuations is a central issue in the current research field.

One of the most important factors relating both blinking and SD phenomena is the Coulomb interaction between optically excited electron-hole pairs and additional charges both inside and outside the NCs. When an additional charge is located inside the NC, optical excitation leads to the formation of charged excitons. This three-body excitonic complex often results in the formation of gray states with intermediate PL intensities, which appear during blinking [12-19]. On the contrary, when additional charges are located outside the NC, their Coulomb potential induces an electric field onto the crystal, which in turn causes a QCSE on the electron-hole pairs in the crystal and results in changes in the transition energies [9,21-24]. Both charges inside and outside the NC affect the PL of single NCs, causing blinking and SD, and thus it is important to elucidate them for the deep understanding of the mechanisms of PL instabilities. However, their mechanisms and the relation between these two phenomena remain unclear, even when both phenomena influence the PL properties of a single NC. This means that, by performing experimental separation of PL signals into the different kinds of excitons - neutral, charged, and QCSE-modified neutral excitons-a better understanding of the PL instability of single NCs can be developed.
In this work, we study SD characteristics of neutral and charged exciton transitions on single CdSe/ZnS NCs. The two contributions are separated by analysis on simultaneously measured PL spectra and lifetimes. The origin of the SD of the neutral exciton transitions is attributed to the QCSE, which causes an increase in the radiative recombination lifetime as a result of a reduction in electron-hole overlap. In addition, we find that the charged excitons are also influenced by the QCSE, resulting in the simultaneous change of radiative and nonradiative recombination lifetime.

\section{EXPERIMENT}

The CdSe/ZnS NCs (QDot605; Invitrogen), diluted in toluene with poly(methyl methacrylate), were first spin coated on a cover glass. The positions of the single NCs were monitored using an electron-multiplying charge-coupled device (EMCCD) camera (ProEM; Roper scientific). For the PL measurements on single NCs, the samples were excited using picosecond light pulses. We set the excitation wavelength at $530 \mathrm{~nm}$, which was obtained by introducing white-light pulses from a supercontinuum light source with a pulse picker (SC400-PP; Fianium) into a monochromator with a focal length of $25 \mathrm{~cm}$. The operating frequency of the pulse picker was set to $5 \mathrm{MHz}$. For the NC excitation and emitted PL detection, an oil-immersion objective lens with a numerical aperture of 1.30 (RMS100X-PFO; Olympus) was used.

To achieve simultaneous measurements of the PL spectra and lifetimes, the PL from single NCs was detected for a period of $100 \mathrm{~s}$ using the optical geometry illustrated in Fig. 1(a). The spectra were recorded using a monochromator with a focal length of $30 \mathrm{~cm}$ equipped with a liquid-nitrogen-cooled CCD camera. The lifetimes were then determined from the decay curves recorded using an avalanche photodiode (id100-50; IDQ) and a time-correlated single-photon counting (TCSPC) board (SCP-130EM; Becker \& Hickl GmbH). We also used an additional avalanche photodiode, with which we could conduct photon-correlation measurements. An example of these photon-correlation measurements is shown in Fig. 1(b), which was recorded under an excitation power at $3.7 \mu \mathrm{W}$. The large ratio of coincidence counts between the center peak at $0 \mathrm{~ns}$ and the side peaks confirms that this NC emits PL under 

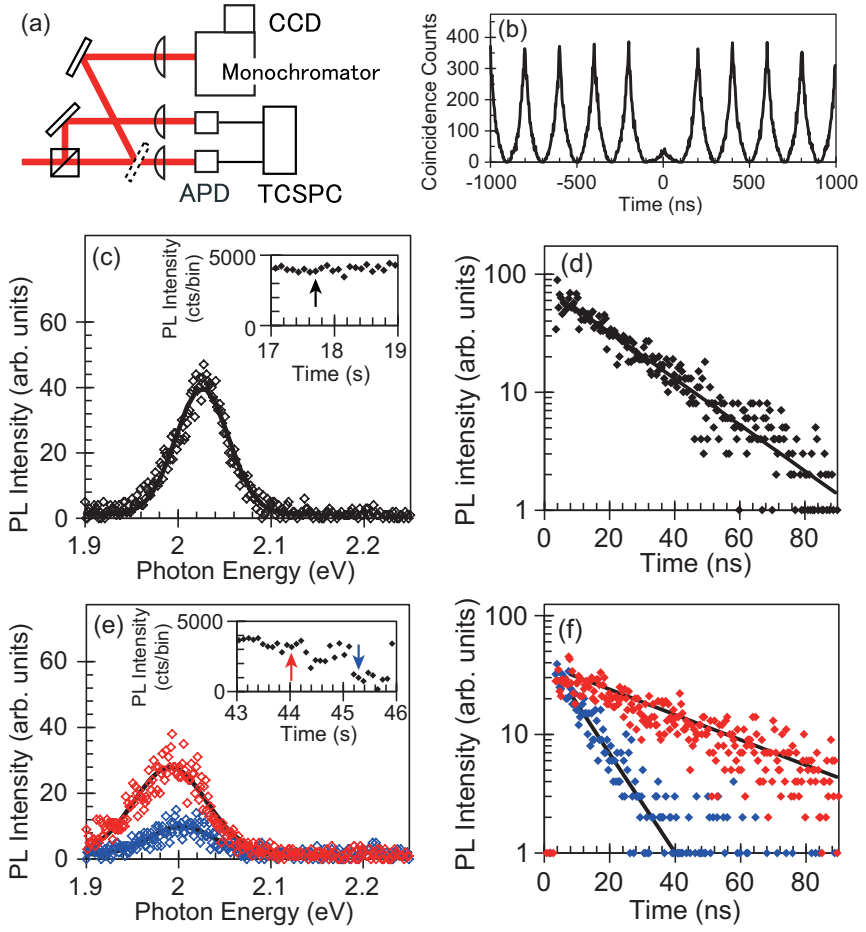

FIG. 1. (Color online) (a) Detection geometry to record PL spectra and lifetime simultaneously. (b) A result of photon-correlation measurement on a single NC. (c) PL spectrum obtained at $17.7 \mathrm{~s}$. The time trace of PL intensity at $17-19 \mathrm{~s}$ is plotted in the inset. A result of Gaussian fitting is shown by solid line. (d) Corresponding PL decay curve obtained at $17.7 \mathrm{~s}$. A result of exponential fitting is shown by solid line. (e), (f) PL spectra and decay curves obtained at $44.0 \mathrm{~s}$ (red) and $45.3 \mathrm{~s}$ (blue). The time trace of PL intensity at $43-46 \mathrm{~s}$ is plotted in the inset of (e).

a single-photon generation regime and, therefore, the $\mathrm{NC}$ is truly a single particle.

\section{RESULTS AND DISCUSSION}

First, we demonstrate the experimental results obtained under an excitation power of $2.6 \mu \mathrm{W}$. To record the PL spectra, the integration time was set to $50 \mathrm{~ms}$. Figure 1(c) is a PL spectrum obtained at $17.7 \mathrm{~s}$. The inset shows the time trace of the PL intensity recorded on the TCSPC board from 17 to $19 \mathrm{~s}$ with a binning time of $\sim 90 \mathrm{~ms}$. The solid line represents a Gaussian function fitted to the PL spectrum. The peak photon energy and the linewidth were found to be 2.026 and $0.04 \mathrm{eV}$, respectively. Figure 1(d) shows the corresponding decay curve obtained on the TCSPC board with a binning time of $\sim 90 \mathrm{~ms}$. The total photon count was 3853 counts (cts)/bin. The solid line shows a single exponential function fitted to the experimental data and the PL lifetime was found to be $22.2 \mathrm{~ns}$. We attribute these signals to neutral exciton emissions because, compared to the signals obtained at other time periods, they display relatively high photon energies, small linewidth, and high PL intensities. In addition, as we will demonstrate in the latter part of this article, this signal dominates the PL signal at lower excitation powers. These PL characteristics can be explained only by the properties of neutral excitons [12-19].
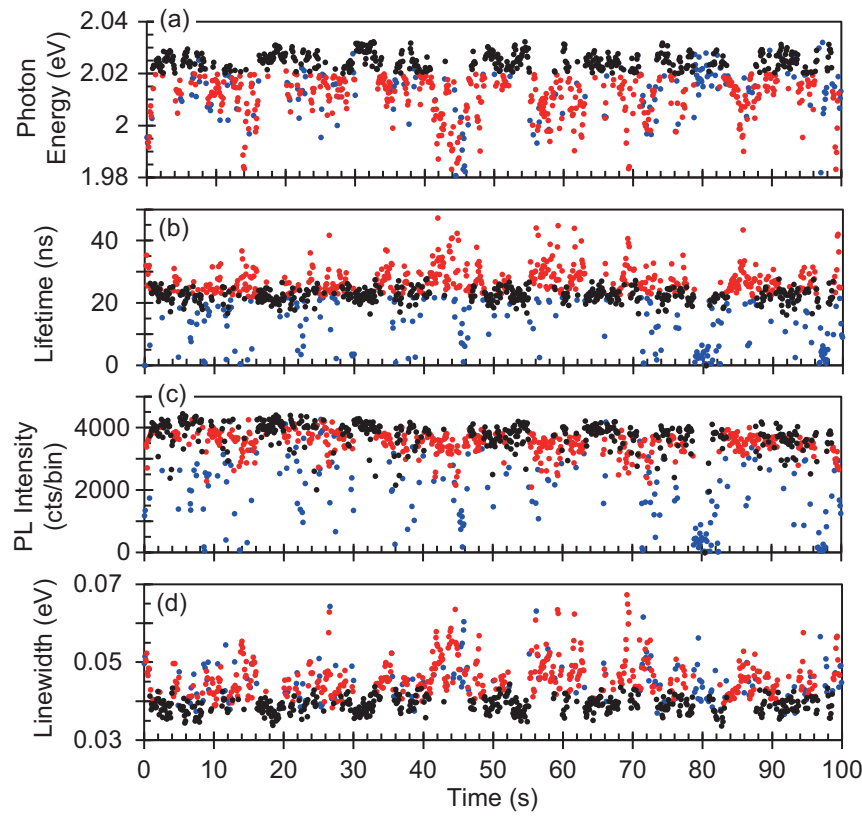

FIG. 2. (Color online) Time traces of (a) photon energy, (b) lifetime, (c) intensity, (d) linewidth at 0-100 s. Each dot is separated into three classes, neutral excitons (black), gray-A (red), and gray-B (blue).

Figure 1(e) shows two PL spectra obtained at $44 \mathrm{~s}$ (red) and $45.3 \mathrm{~s}$ (blue). During the 43-46-s period, as shown in the inset, the time trace of the PL intensity shows time-dependent fluctuations, reflecting the occurrence of PL blinking. Figure 1(f) shows the corresponding decay curves obtained at $44 \mathrm{~s}$ (red) and $45.3 \mathrm{~s}$ (blue). For the data at $44 \mathrm{~s}$, the PL photon energy, linewidth, total photon count, and lifetime were found to be $1.991 \mathrm{eV}, 0.055 \mathrm{eV}, 3156 \mathrm{cts} / \mathrm{bin}$, and $40.9 \mathrm{~ns}$, respectively while, for the data recorded at $45.3 \mathrm{~s}$, they were found to be $2.003 \mathrm{eV}, 0.048 \mathrm{eV}, 978 \mathrm{cts} / \mathrm{bin}$, and $10.4 \mathrm{~ns}$, respectively. Since the PL intensities at 3156 and $978 \mathrm{cts} / \mathrm{bin}$ are smaller than those of the neutral excitons at $3853 \mathrm{cts} / \mathrm{bin}$, we tentatively attribute them to a gray-A state and a gray-B state, respectively. By comparison with the lifetimes of neutral excitons at $22.2 \mathrm{~ns}$, the gray-A state and gray-B state are characterized by the long and short lifetime, respectively.

In order to separate all the signals obtained during a recording period of $100 \mathrm{~s}$ into three classes of neutral excitons, gray-A, and gray-B states, we set criteria considering PL photon energies and lifetimes. Figures 2(a) and 2(b) show the time traces of the PL photon energies and lifetimes, which were determined by Gaussian fitting to the PL spectra and by single-exponential fitting to the PL decay curve, respectively. The black, red, and blue dots correspond to the periods for the neutral excitons, gray-A, and gray-B states, respectively, which were separated using the following procedure. First, we attributed the data exhibiting photon energies larger than $2.02 \mathrm{eV}$ and lifetimes of $22 \pm 6 \mathrm{~ns}$ to the neutral exciton dominant periods. Then, we attribute lifetimes longer (shorter) than 22 ns to the gray-A (gray-B) dominant periods. Through this procedure, it is possible to separate the gray-A and gray-B signals, even though both states show redshifts of almost the same order $(0-0.04 \mathrm{eV})$ compared 
to the neutral excitons, as shown in Fig. 2(a). In Figs. 2(c) and 2(d), the PL intensities and PL linewidths are plotted using three colors to represent the three different classes. The PL intensities of the neutral excitons, gray-A, and gray-B states were at $3500 \pm 1000,3000 \pm 1000$, and ranging from 0 to$3500 \mathrm{cts} / \mathrm{bin}$, respectively. The linewidths of the neutral exciton signals were $0.04 \pm 0.005 \mathrm{eV}$, while those of the gray-A and gray-B states were $0.045 \pm 0.01 \mathrm{eV}$. The periods dominated by neutral excitons plotted in Figs. 2(c) and 2(d) are characterized by large PL intensities and small linewidths, compared to other periods. This feature of neutral excitons agrees with the previous results showing large PL intensities [12-19] and small linewidths $[6-9,20-25]$ for neutral excitons. Thus, the current threshold values of $2.02 \mathrm{eV}$ and $22 \pm 6 \mathrm{~ns}$ are suitable to differentiate the neutral exciton and the other gray states.

To reveal the differences of the above three states more clearly, we show the relationships between the simultaneously measured PL spectra and lifetimes. Figure 3(a) shows photon energy against PL lifetime, and the black, red, and blue dots correspond to the neutral excitons, gray-A, and gray-B states, respectively. Interestingly, with regards to the gray-A signal, the lifetime is extended as the photon energy decreases. On the other hand, for the gray-B signal, no such clear relationship was observed. Figure 3(b) shows the relationship between PL lifetime and intensity, which is known as the fluorescence lifetime-intensity distribution (FLID) plot [14-17,19]. In the case of the gray-A signal, the intensity decreases slightly as the PL lifetime increases while for the gray-B signal, the intensity decreases and approaches zero as the PL lifetime decreases. Figure 3(c) shows the relationship between the PL photon energies and intensities. With regards to the gray-A signal, the intensity decreases with the PL photon energy, while such clear relationships were not observed for the gray-B signal.

We attribute the origin of the gray-A signal (red dots) to the neutral excitons modified by the QCSE (QCSE-modified excitons). An example of the wave function of an electron and that of a hole for QCSE-modified excitons is illustrated in Fig. 3(d), and can be compared with those for neutral excitons with no electric fields given in Fig. 3(e). Generally, the Stark effect results in the redshift of the PL photon energy due to the change of energy level of the electron and hole ground states. At the same time, the radiative recombination lifetime $\left(\tau_{\mathrm{R}}\right)$ increases, since the splitting of the electron and hole wave functions reduces the overlap integral between them [28]. The change in $\tau_{\mathrm{R}}$ affects the PL lifetime $(\tau)$, which is expressed in the form of $1 / \tau=\left(1 / \tau_{\mathrm{R}}\right)+\left(1 / \tau_{\mathrm{NR}}\right)$, where $\tau_{\mathrm{NR}}$ is the nonradiative recombination lifetime. It also influences the PL quantum efficiency (QY), which is proportional to the $\mathrm{PL}$ intensity, since $\mathrm{QY}$ is expressed as $\mathrm{QY}=\tau / \tau_{\mathrm{R}}$. These equations mean that, when the QCSE causes an increase in $\tau_{R}$, which occurs with the redshift of the PL photon energy, it results in an increase of $\tau$ and a decrease in QY. These relationships between PL photon energy, $\tau$, and the QY of the model are consistent with the experimental results illustrated in Figs. 3(a)-3(c), showing the clear relationships between PL photon energies, lifetimes, and PL intensities. This agreement between the experimental results and the model confirm our interpretation of the gray-A signal as being due to QCSEmodified excitons. Thus, we can conclude that the origin of the SD of the neutral exciton transitions is the QCSE, which
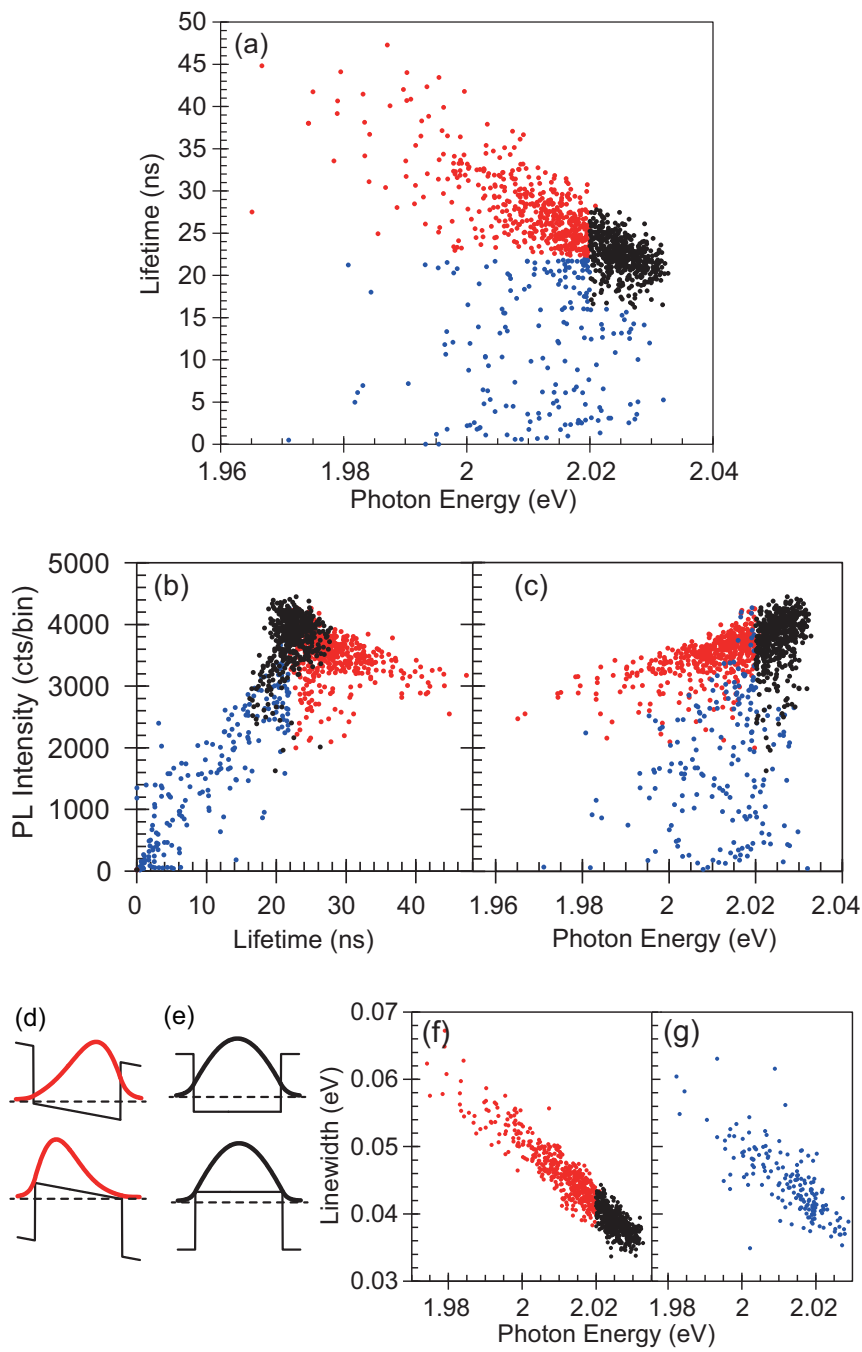

FIG. 3. (Color online) Two-dimensional maps to represent the relationships between (a) photon energy and lifetime, (b) lifetime and intensity, (c) photon energy and intensity. Red and blue dots are attributed to signal of the QCSE-modified excitons and of the charged excitons. Wave functions of electron and hole illustrated for (d) QCSE-modified excitons and (e) neutral excitons without electric field. (f, g) Relationship between photon energy and linewidth for neutral excitons with and without QCSE (f) and for charged excitons (g).

causes an increase in the radiative recombination lifetime due to the reduction of the electron-hole overlap.

With regards to the gray-B signal (blue dots), we attribute its origin to the charged excitons. This assignment is natural since earlier works have also reported similar results concerning the decrease of both PL intensity and lifetime during blinking, and have attributed this effect to charged excitons [12-19]. According to these studies, in the charged exciton states, $\tau_{\mathrm{NR}}$ is shortened because of the presence of an additional electron, which causes an Auger recombination process. In Figs. 3(f) and $3(\mathrm{~g})$, we plot the relationships between PL photon energies and linewidth for neutral excitons (black dots) and QCSEmodified neutral excitons (red dots) and those for charged excitons (blue dots). Interestingly, the clear relationships that exist between them, showing large linewidths for small photon 


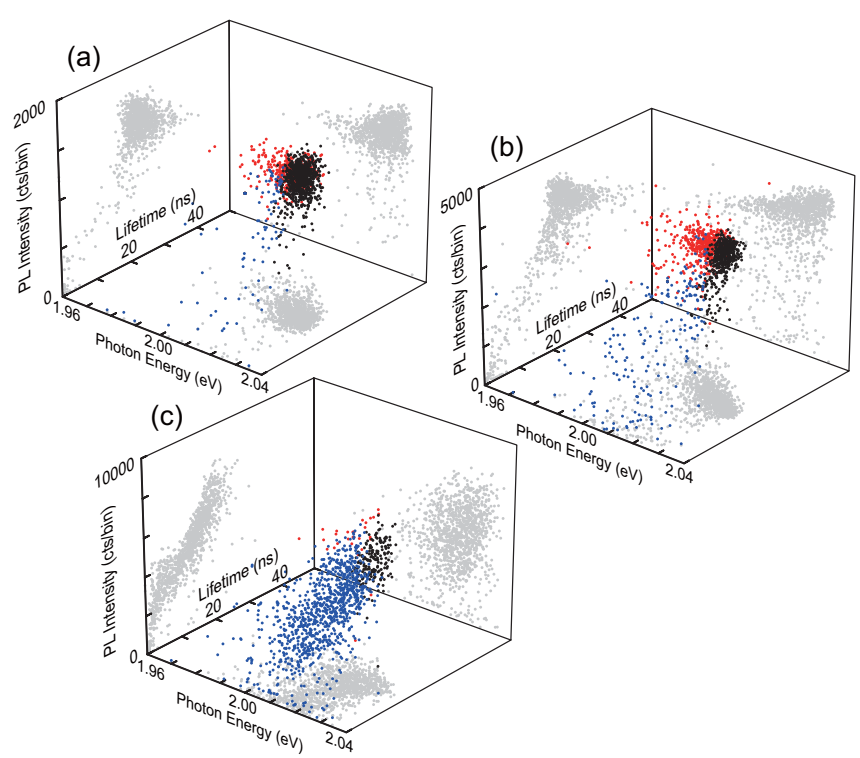

FIG. 4. (Color online) Three-dimensional plots to represent the relationships between photon energy, lifetime, and intensity measured under the elevated excitation power at (a) 0.8 , (b) 2.6 , and (c) $6.6 \mu \mathrm{W}$.

energies, were observed for both QCSE-modified neutral excitons and charged excitons. According to early studies, the increase in linewidth at small photon energies can be explained by the QCSE as it causes electric-field-dependent fluctuations in the PL photon energies [6,24,29]. The fact that this relationship was observed for charged excitons means that the SD of the charged exciton transitions is also influenced by the QCSE. From these results, we conclude that the QCSE causes SD of not only neutral exciton transitions but also charged exciton transitions in single NCs.

The above conclusion means that, for single NCs exhibiting PL fluctuations in the form of blinking and SD, both $\tau_{\mathrm{R}}$ and $\tau_{\mathrm{NR}}$ can change through QCSE and charging. This fact is very important to the understanding of the fundamental nature of PL fluctuations in single NCs. In fact, recent experiments have reported observations of simultaneous changes in $\tau_{\mathrm{R}}$ and $\tau_{\mathrm{NR}}$ during blinking on $\mathrm{CdSe} / \mathrm{CdS} \mathrm{NCs}[15,16]$. The results in two papers indicated that, when $\tau_{\mathrm{NR}}$ decreases due to the charging effect, $\tau_{\mathrm{R}}$ also increases or decreases, hence exhibiting unique relationships between QY and $\tau$. The source of the change in $\tau_{\mathrm{R}}$ can be the activation of an additional excited-state relaxation process [15] or Coulomb interaction in the charged particles [16]. We believe that the QCSE is also one of the possible sources of the changes in $\tau_{\mathrm{R}}$ during blinking, as has been observed for neutral and charged excitons in our experiment.

Let us finally examine how the QCSE is caused during PL blinking. To achieve this, we will discuss additional experimental results obtained at two different excitation densities. In order to visualize the change in the signal, we here introduce three-dimensional (3D) plots of the relationships between the PL intensities, photon energies, and lifetimes. Figure 4(a) shows the 3D view of the results obtained under an excitation power of $0.8 \mu \mathrm{W}$. The black, red, and blue plots correspond to the signals originating from neutral excitons, QCSE-modified excitons, and charged excitons, respectively.
The gray dots correspond to the projections onto each plane forming the 3D space. Figures 4(b) and 4(c) show the results obtained at an excitation power of $2.6 \mu \mathrm{W}$ [which we have already demonstrated in Figs. 3(a)-3(c)] and those recorded at $6.6 \mu \mathrm{W}$, respectively. The classification of the three states was conducted using the same procedure outlined for the results previously discussed (at $2.6 \mu \mathrm{W}$ ). At $0.8 \mu \mathrm{W}$, the neutral exciton signal is dominant, while the occurrences of QCSE-modified excitons and charged excitons are small. However, at $2.6 \mu \mathrm{W}$, the occurrences of both QCSE-modified excitons and charged excitons have increased. At $6.6 \mu \mathrm{W}$, interestingly, the charged exciton signal becomes dominant, while the occurrences of neutral excitons and QCSE-modified excitons have almost disappeared. It is noticeable that the data obtained at $6.6 \mu \mathrm{W}$ show continuous distribution at wide ranges of lifetime and intensity. According to the early works reporting similar results, this could be explained by time-dependent charge migration [29]. We consider that the rapid fluctuation of environment conditions, which results in the fast SD observed in some systems [30,31], could be also one of the origins of the continuous distribution.

The fact that the signal is dominated by the neutral excitons at low excitation powers means that there is no charge either inside or outside the sample under the weak excitation condition. The appearance of QCSE-modified excitons on this NC means that, at higher excitation powers, the excited electron-hole pair sometimes separates and both charges are trapped at various locations in the NC. This is because, if one of the two charges is trapped and the other remains untrapped, this results in the formation of charge excitons. There are some possible locations for the trap state, such as a surrounding matrix [32], ligand [24], surface trap state [9], or interface between the CdSe core and $\mathrm{ZnS}$ shell [29]. The positions of the trapped charges are an important issue in the current research, and thus it should be explored through further investigation on various systems, such as NCs with unique morphologies [33].

\section{CONCLUSION}

In summary, through analysis on simultaneously measured PL spectra and lifetimes on single CdSe/ZnS NCs exhibiting blinking, we distinguished SD of neutral exciton transitions from that of charged exciton transitions. The PL of neutral excitons is found to exhibit a unique SD accompanied by changes of radiative recombination lifetime, which can be explained by the effect of QCSE. We found that the SD of charged exciton transitions is also influenced by the QCSE, resulting in the simultaneous changes of both $\tau_{\mathrm{R}}$ and $\tau_{\mathrm{NR}}$. These results on neutral and charged excitons indicate that the QCSE plays an essential role in PL blinking process. Our findings constitute a fundamental explanation of the mechanisms of the SD and blinking PL fluctuations, and are important for realizing stable single-photon emitters.

\section{ACKNOWLEDGMENT}

Part of this work was supported by KAKENHI (Grant No. 25247052) and JST-CREST. 
[1] J. Cui, A. P. Beyler, T. S. Bischof, M. W. B. Wilson, and M. G. Bawendi, Chem. Soc. Rev. 43, 1287 (2014).

[2] M. J. Fernée, P. Tamarat, and B. Lounis, Chem. Soc. Rev. 43, 1311 (2014).

[3] S. A. Empedocles, D. J. Norris, and M. G. Bawendi, Phys. Rev. Lett. 77, 3873 (1996).

[4] M. Nirmal, B. O. Dabbousi, M. G. Bawendi, J. J. Macklin, J. K. Trautman, T. D. Harris, and L. E. Brus, Nature 383, 802 (1996).

[5] M. Kuno, D. P. Fromm, H. F. Hamann, A. Gallagher, and D. J. Nesbitt, J. Chem. Phys. 112, 3117 (2000).

[6] S. A. Empedocles and M. G. Bawendi, Science 278, 2114 (1997).

[7] S. A. Empedocles and M. G. Bawendi, J. Phys. Chem. B 103, 1826 (1999).

[8] R. G. Neuhauser, K. T. Shimizu, W. K. Woo, S. A. Empedocles, and M. G. Bawendi, Phys. Rev. Lett. 85, 3301 (2000).

[9] J. Müller, J. M. Lupton, A. L. Rogach, J. Feldmann, D. V. Talapin, and H. Weller, Phys. Rev. Lett. 93, 167402 (2004).

[10] S. Rosen, O. Schwartz, and D. Oron, Phys. Rev. Lett. 104, 157404 (2010).

[11] J. Zhao, G. Nair, B. R. Fisher, and M. G. Bawendi, Phys. Rev. Lett. 104, 157403 (2010)

[12] P. Spinicelli, S. Buil, X. Quélin, B. Mahler, B. Dubertret, and J.-P. Hermier, Phys. Rev. Lett. 102, 136801 (2009).

[13] D. E. Gómez, J. V. Embden, P. Mulvaney, M. J. Fernée, and H. Rubinsztein-Dunlop, ACS Nano 3, 2281 (2009).

[14] N. Amecke and F. Cichos, J. Lumin. 131, 375 (2011).

[15] C. Galland, Y. Ghosh, A. Steinbrück, M. Sykora, J. A. Hollingsworth, V. I. Klimov, and H. Htoon, Nature 479, 203 (2011).

[16] C. Galland, Y. Ghosh, A. Steinbrück, J. A. Hollingsworth, H. Htoon, and V. I. Klimov, Nat. Commun. 3, 908 (2012).

[17] F. T. Rabouw, P. Lunnemann, R. J. A. van Dijk-Moes, M. Frimmer, F. Pietra, A. F. Koenderink, and D. Vanmaekelbergh, Nano Lett. 13, 4884 (2013).
[18] N. Yoshikawa, H. Hirori, H. Watanabe, T. Aoki, T. Ihara, R. Kusuda, C. Wolpert, T. K. Fujiwara, A. Kusumi, Y. Kanemitsu, and K. Tanaka, Phys. Rev. B 88, 155440 (2013).

[19] T. Ihara, R. Sato, T. Teranishi, and Y. Kanemitsu, Phys. Rev. B 90, 035309 (2014).

[20] T. Plakhotnik, M. J. Fernée, B. Littleton, H. Rubinsztein-Dunlop, C. Potzner, and P. Mulvaney, Phys. Rev. Lett. 105, 167402 (2010).

[21] M. J. Fernée, B. Littleton, T. Plakhotnik, H. Rubinsztein-Dunlop, D. E. Gómez, and P. Mulvaney, Phys. Rev. B 81, 155307 (2010)

[22] M. J. Fernée, T. Plakhotnik, Y. Louyer, B. N. Littleton, C. Potzner, P. Tamarat, P. Mulvaney, and B. Lounis, J. Phys. Chem. Lett. 3, 1716 (2012).

[23] K. W. Park, Z. Deutsch, J. J. Li, D. Oron, and S. Weiss, ACS Nano 6, 10013 (2012).

[24] D. Braam, A. Mölleken, G. M. Prinz, C. Notthoff, M. Geller, and A. Lorke, Phys. Rev. B 88, 125302 (2013).

[25] A. P. Beyler, L. F. Marshall, J. Cui, X. Brokmann, and M. G. Bawendi, Phys. Rev. Lett. 111, 177401 (2013).

[26] P. Michler, A. Imamoglu, M. D. Mason, P. J. Carson, G. F. Strouse, and S. K. Buratto, Nature 406, 968 (2000).

[27] B. Lounis and M. Orrit, Rep. Prog. Phys. 68, 1129 (2005).

[28] H.-J. Polland, L. Schultheis, J. Kuhl, E. O. Göbel, and C. W. Tu, Phys. Rev. Lett. 55, 2610 (1985).

[29] K. Zhang, H. Chang, A. Fu, A. P. Alivisatos, and H. Yang, Nano Lett. 6, 843 (2006).

[30] G. Sallen, A. Tribu, T. Aichele, R. André, L. Besombes, C. Bougerol, M. Richard, S. Tatarenko, K. Kheng, and J.-Ph. Poizat, Nat. Photonics 4, 696 (2010).

[31] L. Coolen, X. Brokmann, P. Spinicelli, and J.-P. Hermier, Phys. Rev. Lett. 100, 027403 (2008).

[32] D. E. Gómez, J. V. Embden, and P. Mulvaney, Appl. Phys. Lett. 88, 154106 (2006).

[33] N. J. Borys, M. J. Walter, J. Huang, D. V. Talapin, and J. M. Lupton, Science 330, 1371 (2010). 